\title{
SEMIGROUPS OF TRUNCATED TOEPLITZ OPERATORS
}

\author{
Ameur Yagoub And MOHAMEd ZarRabi
}

Abstract. We characterise the one parameter families of truncated Toeplitz operators that are semigroups, uniformly continuous semigroups and $C_{0}$-semigroups of contractions. We also study their generators.

Mathematics subject classification (2010): 47B35, 47D03, 30H10, 47B44.

Keywords and phrases: Model space, truncated Toeplitz operators, Semigroups.

\section{REFERENCES}

[1] I. Chalendar, D. Timotin, Commutation relations for truncated Toeplitz operators, Operators and Matrices 8, no. 3 (2014), 877-888.

[2] D. N. CLARK, One dimensional perturbations of restricted shifts, J. Analyse Math. 25 (1972), 169191.

[3] J. B. Conway, A course in Functional Analysis, Second edition, Graduate Text in Mathematics, 96. Springer-Verlag, New York, 1990.

[4] R. B. Crofoot, Multipliers between invariant subspaces of the backward shift, Pacific J. Math. 166 (1994), no. 2, 225-246.

[5] K. ENGEL And R. NAGEL, One-Parameter Semigroups for Linear Evolution Equations, Graduate Texts in Mathematics, Springer, New York, 1995.

[6] S. R. Garcia, M. Putinar, Complex symmetric operators and applications, Trans. Amer. Math. Soc. 358 (2006), 1285-1315.

[7] S. R. GARCIA, W. T. Ross, Recent progress on truncated Toeplitz operators, in: J. Mashreghi, E. Fricain (Eds.), Blaschke Products and Their Applications, in: Fields Inst. Commun., vol. 65, Springer, New York, (2013), 275-319.

[8] R. V. KAdison, J. R. Ringrose, Fundamentals of the theory of operator algebras. Vol. I. Elementary theory, Graduate Studies in Mathematics, 15. American Mathematical Society, Providence, RI, 1997.

[9] A. PAZY, Semigroups of Linear Operators and Applications to Partial Differential Equations, Springer-Verlag, New York, 1983.

[10] D. SARASON, Algebraic properties of truncated Toeplitz operators, Oper. Matrices 1 (2007), 491-526.

[11] D. SARASON, Unbounded operators commuting with restricted backwards shifts, Oper. Matrices, 2 (2008), 583-601.

[12] D. SARASON, Unbounded Toeplitz operators, Integral Equations Oper. Theory 61 (2008), 281-298.

[13] N. A. SEDLOCK, Algebras of truncated Toeplitz operators, Oper. Matrices 5 (2011), 309-326.

[14] N. A. SEDlock, Properties of Truncated Toeplitz Operators, ProQuest LLC, Ann Arbor, MI, 2010. Ph.D. thesis, Washington University in St. Louis.

[15] S.M. SEUBERT, Dissipative compressed Toeplitz operators on shift co-invariant subspaces, Houston J. Math., 32, no. 1 (2006), 277-292.

[16] S.M. SEUBERT, Semigroups of operators on quotient spaces of $H^{2}$, J. Math. Anal. Appl. 167 (1992) 289-298.

[17] S. M. Seubert, Unbounded dissipative compressed Toeplitz operators, J. Math. Anal. Appl. 290 (2004), 132-146.

[18] D. SuÁrEZ, Closed commutants of the backwards shift operator, Pacific J. Math., 179 (1997), $371-$ 396. 